BMJ Open

Diabetes

Research

\& Care

\title{
Long-term tracking of fasting blood glucose variability and peripheral artery disease in people without diabetes
}

\author{
Xiu-Ting Sun (1D , ${ }^{1,2}$ Cheng Zeng, ${ }^{1}$ Shao-Zhao Zhang, ${ }^{1,2}$ Hui-Min Zhou, ${ }^{1,2}$ \\ Xiang-Bin Zhong, ${ }^{1,2}$ Zhen-Yu Xiong, ${ }^{1,2}$ Da-Ya Yang, ${ }^{1,2}$ Yue Guo, ${ }^{1,2}$ \\ Xiao-Dong Zhuang, ${ }^{1,2,3}$ Xin-Xue Liao ${ }^{1,2}$
}

To cite: Sun X-T, Zeng C, Zhang S-Z, et al. Long-term tracking of fasting blood glucose variability and peripheral artery disease in people without diabetes. BMJ Open Diab Res Care 2020;8:e000896. doi:10.1136/ bmjdrc-2019-000896

- Supplemental material is published online only. To view, please visit the journal online (http://dx.doi.org/10.1136/ bmjdrc-2019-000896).

X-TS and CZ contributed equally.

Received 11 September 2019 Revised 10 March 2020 Accepted 12 March 2020

\section{Check for updates}

\section{(C) Author(s) (or their} employer(s)) 2020. Re-use permitted under CC BY-NC. No commercial re-use. See rights and permissions. Published by BMJ.

\section{${ }^{1}$ Cardiology, Sun Yat-sen} University First Affiliated Hospital, GuangZhou, China ${ }^{2} \mathrm{NHC}$ Key Laboratory of Assisted Circulation (Sun Yatsen University), GuangZhou, China

${ }^{3}$ Center for Information Technology \& Statistics, Sun Yat-sen University First Affiliated Hospital, GuangZhou, China

Correspondence to Dr Xiao-Dong Zhuang; zhuangxd3@mail.sysu.edu.cn and Professor Xin-Xue Liao; liaoxinx@mail.sysu.edu.cn

\section{ABSTRACT}

Introduction Long-term changes of fasting blood glucose (FBG) in relation to lower-extremity peripheral artery disease (lower-extremity PAD) in people without diabetes has barely been reported. Our study aimed to investigate the association between FBG variability and the incidence of lower-extremity PAD in people without diabetes.

Research design and methods We included 7699 participants without prior lower-extremity PAD and diabetes from the Atherosclerosis Risk in Communities study in the final analysis. At least two measurements of FBG were required during follow-up. Variability of FBG was identified using SD, coefficient of variation (CV), variability independent of the mean (VIM) and average real variability. Lower-extremity PAD was defined as an ankle brachial index $<0.9$, or hospitalization with a lower-extremity PAD diagnosis. Cox regression model was used to calculate HR for incidence of lower-extremity PAD and FBG variability. Results During a median follow-up of 19.5 years, 504 (6.5\%) lower-extremity PAD events were observed, $54.4 \%$ $(n=274)$ were male, and $17.5 \%(n=88)$ were AfricanAmerican. FBG variability was positively associated with incident lower-extremity PAD, with a linear relationship. HRs for CV and VIM were 1.015 (95\% Cl: 1.001 to 1.03; $\mathrm{p}=0.023$ ), and 1.032 (95\% Cl: 1.004 to $1.06 ; p=0.022$ ) for lower-extremity PAD, respectively. Participants in the lowest quartile of $\mathrm{CV}$ were at lower lower-extremity $\mathrm{PAD}$ risk compared with the highest ones (HR: $1.499,95 \% \mathrm{Cl}$ : 1.16 to $1.938 ; p=0.002$ ).

Conclusions Higher FBG variability was independently associated with increased prevalence of lower-extremity PAD in people without diabetes.

Trial registration number NCT00005131.

\section{INTRODUCTION}

Lower-extremity peripheral artery disease (lower-extremity PAD) is a severe global health problem, especially in high-income countries during the past few decades. ${ }^{1}$ Earlier studies have illustrated that many traditional risk factors such as hypertension (HT), diabetes and hyperlipidemia can accelerate the development of lower-extremity PAD. ${ }^{2-4}$ Our previous studies further demonstrated that new factors such as serum magnesium, cadmium and $\mathrm{C}$ reactive protein were

\section{Significance of this study}

What is already known about this subject?

- Fasting blood glucose (FBG) was associated with risk of vascular disease in people without diabetes.

- Higher variability of FBG increased the risk of cardiovascular events and mortality.

What are the new findings?

- In a population without diabetes, FBG variability was positively associated with the risk of lowerextremity peripheral artery disease (PAD), with a linear relationship.

How might these results change the focus of research or clinical practice?

- Our study suggested that glycemic changes should be emphasized even in people without diabetes.

- Additional studies are needed to confirm our findings and explore the mechanisms for the relation between FBG variability and lower-extremity PAD.

associated with the incidence of lowerextremity PAD.${ }^{5}$ Hyperglycemia is a known risk factor for cardiovascular disease (CVD). However, less attention was paid to the role of normal blood glucose in the process of lowerextremity PAD. Recent studies showed fasting blood glucose (FBG) was associated with the risk of atherosclerosis (AS) in people without diabetes. ${ }^{78}$ Thus, exploring the association between FBG with lower-extremity PAD in people without diabetes may help clinicians to better understand lower-extremity PAD.

Tracking may be defined as the longitudinal stability of a certain risk factor or the predictability of a measurement early in life for values later in life, which is a more effective way to conduct studies. ${ }^{9}$ Long-term follow-up of risk factors could provide more information than just one-time measurement. High variability of FBG and hemoglobin A1c (HbAlc) could increase the risk of cardiovascular events and mortality. ${ }^{10} 11$ We also found that higher 
FBG variability in young adulthood was associated with lower midlife hippocampal integrity and volume in our recent study. ${ }^{12}$ Actually, long-term changes of risk factors in people without diagnosis of diseases. Changes in insulin, blood pressure (BP) and blood lipids over time had an impact on health many years before diagnosis of diseases. ${ }^{13-16}$ Norby et $^{17} \mathrm{l}^{17}$ studied the trajectories of atrial fibrillation (AF) risk factors for over 25 years, and found that patients with $\mathrm{AF}$ increased the prevalence of risk factors 15 years before AF diagnosis. Therefore, studying the trajectories of risk factors is very important for the prevention and treatment of diseases. So far, few studies focused on the association between long-term tracking of FBG and lower-extremity PAD.

The Atherosclerosis Risk in Communities (ARIC) study is a large population-based study with an over 25-year follow-up, which is proper for us to study long-term trajectories of risk factors. Thus, we used data from ARIC to investigate the relation between FBG variability and incident lower-extremity PAD.

\section{METHODS}

\section{Study population}

A total of 15792 participants were recruited from four US communities in the ARIC study. Participants in ARIC were re-examined four times after the first screen (baseline, visit 1, 1987-1989), then followed by four examinations (visit 2 in 1990-1992, visit 3 in 1993-1995, visit 4 in 1996-1998 and visit 5 in 2011-2013). All participants provided informed consent before each visit. ${ }^{18}$

The first three measurements of FBG was used to calculate FBG variability, the third visit (visit 3) was defined as baseline in the current study. We excluded those participants with prior lower-extremity PAD (from visit 1 to visit $3 ; \mathrm{n}=1415$ ) at baseline, all diabetes from visit 1 to visit 5 $(n=3304)$, participants with just one-time measurement of FBG ( $n=307)$, those without FBG measurement at visit $3(n=42)$, as well as those with missing covariates $(n=166)$, leaving 7699 participants in the final analysis (online supplemental file 1 ).

\section{Variability of fasting blood glucose}

Blood sample was collected from participants who were asked to fast for 8 hours. Aliquots were stored at $-70^{\circ} \mathrm{C}$ for further analyses. FBG was detected using hexokinase/ glucose-6-phosphate dehydrogenase method. Diabetes mellitus (DM) was defined as FBG level $\geq 126 \mathrm{mg} / \mathrm{dL}$ $(\geq 7 \mathrm{mmol} / \mathrm{L})$, non-fasting glucose level $\geq 200 \mathrm{mg} / \mathrm{dL}$ $(\geq 11.1 \mathrm{mmol} / \mathrm{L})$ or medication use, or self-reported physician diagnosis. Variability of FBG was used to investigate trajectory, which was calculated using 1) the coefficient of variation $(\mathrm{CV})$; 2) the variability independent of the mean (VIM), which was calculated as $100 \times \mathrm{SD} /$ mean $\beta$, where $\beta$ is the regression coefficient based on natural logarithm of SD on natural logarithm of mean; 3) the average real variability (ARV), which was calculated as the absolute difference between successive measurements of FBG, divided by the duration between FBG measurements to create annualized ARV of FBG between all examinations. All the measurements of variability have been previously described. ${ }^{19-21}$

\section{Lower-extremity peripheral artery disease definition}

Individual follow-up for events started from the third visit and continued when lower-extremity PAD occurred or their date of death, or the end of ARIC follow-up, whichever happened first. Lower-extremity PAD was defined as an ankle brachial index (ABI) $<0.9$, or a hospital discharge diagnosis of lower-extremity PAD, peripheral artery revascularization procedure or peripheral artery intervention therapy during follow-up. ${ }^{18}$

$\mathrm{ABI}$ was defined as the ratio of the ankle systolic blood pressure (SBP) to the brachial SBP. Dinamap 1846 automated oscillometric device (Criticon, Tampa, Florida, USA) was used to measure ankle SBP at the posterior tibial artery with the participant prone, and brachial SBP in the right arm with the participant supine. ${ }^{22}$ We used the following International Classification of Diseases, Ninth Revision codes to diagnose lower-extremity PAD: 39.25 (aorto-iliac-femoral bypass), 39.29 (leg bypass surgery), 84.11 (toe amputation), 84.12 (foot amputation), 84.15 (below-knee amputation), 84.17 (above-knee amputation), 38.18 (leg endarterectomy), 440.20 (AS of native arteries of the extremities, unspecified), 440.3 (AS of bypass graft of the extremities), 440.4 (chronic total occlusion of artery of the extremities), 443.81 (peripheral angiopathy in diseases classified elsewhere), 443.9 (claudication, peripheral arterial disease not otherwise specified, peripheral angiopathy not otherwise specified, spasm of artery).

\section{Other variables of interest}

We got information of age, race, sex, smoking and drinking status, education levels and medication use of participants by self-report. SBP $\geq 140 \mathrm{~mm} \mathrm{Hg}$ or diastolic blood pressure (DBP) $\geq 90 \mathrm{~mm} \mathrm{Hg}$ or use of antihypertensive medication, or physician diagnosis were used to define HT. Prevalent coronary heart disease (CHD) was acquired by self-reported history of myocardial infarction, heart surgery, coronary bypass or balloon angioplasty or current medication use.

Body mass index (BMI) was calculated by dividing weight in kilograms by height in meters squared. An estimated glomerular filtration rate (eGFR) was calculated using the four-variable Modification of Diet in Renal Disease equation. ${ }^{23}$ Enzymatic method was used to measure high-density lipoprotein cholesterol (HDL-C), total cholesterol (TC), triglycerides (TG) and Friedewald equation was used to calculate low-density lipoprotein cholesterol (LDL-C). ${ }^{18}$

\section{Statistical analysis}

Baseline characteristics of participants were described by means and proportions. Analysis of variance was used to compare baseline continuous variables and chi-square 
tests for categorical variables. Tests were two-sided, and a $p$ value of $<0.05$ was considered statistically significant.

We modeled each measure FBG variability (SD, CV, VIM and ARV) with the lowest quartile serving as the reference. Linear trends for all continuous variables were tested through the use of restricted cubic splines. In the spline models, CV of FBG as restricted quartile splines with knots at the 5th, 50th and 95th percentiles of its distribution to provide the relationship between CV of FBG and lower-extremity PAD. Cox proportional hazard regression models were used to calculate $\mathrm{HR}$ and $95 \%$ CIs between FBG variability and time to lower-extremity PAD. Model 1 included adjustment for baseline age, sex, race, education levels, smoking and drinking status. Traditional lower-extremity PAD risk factors such as BMI, SBP, DBP, TC, TG, HDL-C, LDL-C, baseline FBG and eGFR were added in model 2. Model 3 further adjusted for prevalent CHD, HT, history of stroke and use of medication.

We performed subgroup analysis stratified by key demographic and clinical subgroups of age ( $<60 \mathrm{vs} \geq 60$ years), gender (female vs male), race (white vs black), smoking status (current vs former), kidney function (eGFR $<60 \mathrm{vs}$ $\geq 60 \mathrm{~mL} / \mathrm{min} / 1.73 \mathrm{~m}^{2}$ ). We also stratified the subgroup analysis by prediabetes status (yes vs no) according to baseline FBG $(<100$ vs $100-126 \mathrm{mg} / \mathrm{dL}(5.6-7.0 \mathrm{mmol} / \mathrm{L}))$. We further used data from CVD (prevalent of CHD, HT or history of stroke) to conduct sensitivity analysis. Also, we conducted sensitivity analysis excluding participants with missing data. We used mean imputation to deal with missing data. To test for differences within the various subgroups, $\mathrm{p}$ values for interaction were derived from multivariable Cox regression models. All analyses were done using SPSS V.22.0 and Stata V.3.0.

\section{RESULTS}

\section{Baseline characteristics stratified by CV of FBG}

Among 7699 participants included in our study, 44.7\% were male, $83.1 \%$ were white and the mean \pm SD age was $60 \pm 5.7$ years. The median follow-up was 19.5 years. Table 1 summarizes the baseline characteristics of study population by CV levels. Participants in the highest CV quartiles were black, and had higher BMI, DBP, SBP, baseline FBG, TG, eGFR as well as LDL-C levels compared with the lowest ones. And, people in Q4 were more likely to have suffered from prevalent CHD, HT and stroke. Nevertheless, no significant differences among age, gender, TC, HDL-C and use of aspirin and statin were found.

\section{FBG variability and lower-extremity PAD}

After a medium follow-up of 19.5 years, $504(6.5 \%)$ lowerextremity PAD events were observed, 54.4\% ( $\mathrm{n}=274)$ were male, and $17.5 \%(\mathrm{n}=88)$ were African-American. We found that higher FBG variability was associated with increased incidence of lower-extremity PAD (table 2) (HR: $1.015,95 \% \mathrm{CI}: 1.001$ to $1.03 ; \mathrm{p}=0.022$ ). Spline regression analysis further confirmed that FBG variability was positively associated with the risk of lower-extremity PAD, with a linear relationship (figure 1).

As shown in table 2, after adjusted for multiple adjustment (including age, gender, race, smoking and drinking status, education level, lipids level, BMI, SBP, DBP, baseline FBG, eGFR and prevalent CHD, HT, history of stroke and use of medication), HRs for VIM and ARV were 1.032 (95\% CI: 1.004 to $1.06 ; \mathrm{p}=0.023)$ and $1.016(95 \%$ CI: 1.002 to $1.03 ; \mathrm{p}=0.027$ ) for lower-extremity PAD, respectively. When FBG variability was analyzed as categorical variable, we found that participants in the lowest quartile of $\mathrm{CV}$ were at lower lower-extremity PAD risk compared with the highest ones (HR $=1.499,95 \%$ CI: 1.16 to $1.938 ; \mathrm{p}=0.002 ; \mathrm{p}$ for trend=0.011). And similar results were observed with other measures of FBG variability including VIM and ARV (table 2, $\mathrm{p}$ for trends $<0.05$ ).

\section{Subgroup and sensitivity analysis}

Figure 2 summarizes the results of subgroup and sensitivity analysis in our study. When stratified by age, gender and race, the association between FBG variability and lowerextremity PAD was consistent with our previous findings ( $p$ for interactions $>0.05$ ). Also, no statistically significant interactions were found between FBG variability and smoking status, kidney dysfunction, prevalence of CHD, HT and stroke (all $\mathrm{p}$ for interactions $>0.05$ ).

Participants were stratified with prediabetes status (yes or no) according to baseline FBG. HRs for FBG variability $(\mathrm{CV})$ and incident lower-extremity PAD were 1.017 and 1.006 ( $\mathrm{p}=0.048$ and 0.099$)$. However, no statistically significant interaction was found between those with prediabetes and normoglycemia ( $\mathrm{p}$ for interaction $=0.77$ ) (figure 2).

\section{DISCUSSION}

In our study, we found a positive association between FBG variability and lower-extremity PAD in a large population without diabetes. After multiple adjustment, there was a linear relation between FBG variability and the risk of lower-extremity PAD. Our results provided additional information on the relation of FBG and lower-extremity $\mathrm{PAD}$, highlighting the importance of FBG variability in the development of lower-extremity PAD.

As previous studies demonstrated, blood glucose played a significant role in the process of AS in people with or without diabetes. ${ }^{24} 25$ The Asia Pacific Cohort Studies Collaboration investigated 237468 participants with or without diabetes, found that FBG within normal range was positive continuous associated with the incidence of CVD. FBG lowering down to levels of at least $4.9 \mathrm{mmol} / \mathrm{L}$ could reduce CVD burden. ${ }^{26}$ As changes of risk factors over time were more reliable than just one-time measurement, more and more studies focused on the association between long-term tracking of blood glucose and CVD. ${ }^{27}{ }^{28}$ Ghouse et $a l^{10}$ investigated 6756 participants without diabetes, found that high HbA1c variability was positively related to major adverse cardiovascular events 
Table 1 Baseline characteristics of participants according to quartiles of CV of fasting blood glucose

\begin{tabular}{|c|c|c|c|c|c|}
\hline Characteristics & Q1 & Q2 & Q3 & Q4 & $P$ value \\
\hline CV\% & $\leq 2.8$ & $2.8-4.42$ & $4.42-6.4$ & $>6.4$ & \\
\hline $\mathrm{N}$ & 1917 & 1933 & 1922 & 1927 & \\
\hline Age, years & $60.18 \pm 5.67$ & $60.08 \pm 5.68$ & $60.04 \pm 5.71$ & $60.35 \pm 5.7$ & 0.353 \\
\hline Gender & & & & & 0.124 \\
\hline Female & $1035(54 \%)$ & 1081 (55.9\%) & 1099 (57.2\%) & $1040(54 \%)$ & \\
\hline Male & $882(46 \%)$ & $852(44.1 \%)$ & $823(42.8 \%)$ & $887(46 \%)$ & \\
\hline Race & & & & & $<0.0001$ \\
\hline White & 1681 (87.7\%) & $1662(86 \%)$ & 1591 (82.8\%) & 1463 (75.9\%) & \\
\hline Black & 236 (12.3\%) & $271(14 \%)$ & 331 (17.2\%) & 464 (24.1\%) & \\
\hline \multicolumn{6}{|l|}{ Smoking status } \\
\hline Current & $320(26.8 \%)$ & 317 (23.3\%) & $338(24.1 \%)$ & $410(28.1 \%)$ & $<0.0001$ \\
\hline Former & $790(32.8 \%)$ & 777 (33.6\%) & $770(33 \%)$ & 790 (33.2\%) & 0.854 \\
\hline \multicolumn{6}{|l|}{ Drinking status } \\
\hline Current & 1129 (58.9\%) & $1086(56.2 \%)$ & 1103 (57.4\%) & 996 (51.7\%) & $<0.0001$ \\
\hline Former & $376(19.6 \%)$ & 379 (19.6\%) & $374(19.5 \%)$ & $447(23.2 \%)$ & 0.003 \\
\hline Education level & & & & & $<0.0001$ \\
\hline 1 (basic) & 287 (15\%) & 304 (15.7\%) & $321(16.7 \%)$ & $405(21 \%)$ & \\
\hline 2 (intermediate) & $821(42.8 \%)$ & 819 (42.4\%) & 769 (40\%) & 780 (40.5\%) & \\
\hline 3 (advanced) & $769(40.1 \%)$ & 765 (39.6\%) & $781(40.6 \%)$ & 685 (35.5\%) & \\
\hline BMI, $\mathrm{kg} / \mathrm{m}^{2}$ & $27.16 \pm 4.54$ & $27.07 \pm 4.62$ & $27.39 \pm 4.83$ & $28.34 \pm 5.6$ & $<0.0001$ \\
\hline $\mathrm{SBP}, \mathrm{mm} \mathrm{Hg}$ & $121.62 \pm 18.11$ & $122.29 \pm 18.35$ & $121.71 \pm 18.47$ & $125.41 \pm 19.85$ & $<0.0001$ \\
\hline DBP, mm Hg & $70.73 \pm 10.11$ & $71.04 \pm 9.99$ & $71.13 \pm 10.16$ & $72.2 \pm 10.72$ & $<0.0001$ \\
\hline $\mathrm{FBG}, \mathrm{mg} / \mathrm{dL}$ & $98.05 \pm 8.15$ & $97.17 \pm 9.08$ & $97.54 \pm 10.11$ & $107.6 \pm 40.98$ & $<0.0001$ \\
\hline eGFR, mL/min/1.73 $\mathrm{m}^{2}$ & $68.03 \pm 16.2$ & $67.91 \pm 15.86$ & $69.05 \pm 10.53$ & $70.8 \pm 19.23$ & $<0.0001$ \\
\hline $\mathrm{TC}, \mathrm{mmol} / \mathrm{L}$ & $5.35 \pm 0.95$ & $5.35 \pm 0.92$ & $5.39 \pm 0.96$ & $5.36 \pm 0.99$ & 0.616 \\
\hline $\mathrm{HDL}-\mathrm{C}, \mathrm{mmol} / \mathrm{L}$ & $1.38 \pm 0.46$ & $1.4 \pm 0.48$ & $1.4 \pm 0.46$ & $1.39 \pm 0.51$ & 0.413 \\
\hline LDL-C, $\mathrm{mmol} / \mathrm{L}$ & $3.26 \pm 0.91$ & $3.23 \pm 0.91$ & $3.32 \pm 0.92$ & $3.21 \pm 1.03$ & 0.003 \\
\hline $\mathrm{TG}, \mathrm{mmol} / \mathrm{L}$ & $1.5 \pm 0.79$ & $1.51 \pm 0.82$ & $1.45 \pm 0.75$ & $1.58 \pm 1.09$ & $<0.0001$ \\
\hline Prevalent HT & $583(30.4 \%)$ & $666(34.5 \%)$ & $643(33.5 \%)$ & $832(43.2 \%)$ & $<0.0001$ \\
\hline Prevalent CHD & $116(6.1 \%)$ & $117(6.1 \%)$ & 109 (5.7\%) & $253(13.1 \%)$ & $<0.0001$ \\
\hline Prevalent stroke & $33(1.7 \%)$ & $41(2.1 \%)$ & $59(3.1 \%)$ & $65(3.4 \%)$ & 0.003 \\
\hline Anti-HT medicine use & $548(28.6 \%)$ & $584(30.2 \%)$ & $613(31.9 \%)$ & $815(42.3 \%)$ & $<0.0001$ \\
\hline Aspirin use & $1020(53.2 \%)$ & $1033(53.4 \%)$ & $1031(53.6 \%)$ & $1044(54.2 \%)$ & 0.941 \\
\hline Statin use & 22 (1.1\%) & 14 (0.7\%) & 20 (1\%) & 30 (1.6\%) & 0.103 \\
\hline
\end{tabular}

Values are mean \pm SD or number (\%).

$\mathrm{BMI}$, body mass index; CHD, coronary heart disease; CV, coefficient of variation; DBP, diastolic blood pressure; eGFR, estimated glomerular filtration rate; FBG, fasting blood glucose; HDL-C, high-density lipoprotein cholesterol; HT, hypertension; LDL-C, low-density lipoprotein cholesterol; SBP, systolic blood pressure; TC, total cholesterol; TG, triglycerides.

and all-cause mortality. Echouffo-Tcheugui $e t a l^{11}$ demonstrated that greater variability of FBG was associated with increased mortality risk. However, few studies have considered the long-term glycemic variability of FBG variability in relation to lower-extremity $\mathrm{PAD}$, especially in people within normal FBG range. To expand the findings of previous studies, we examined the influence of glycemic variability among participants without diabetes. In the current study, we used three methods to assess long-term
FBG variability. Consistent with previous studies, our results further demonstrated that higher glycemic variability was positively related to incident lower-extremity PAD in people without diabetes, with a linear relationship ( $p$ for trend $<0.05$ ). Individuals with a higher variability of FBG tended to be black, with higher baseline FBG, $\mathrm{BP}$ and BMI levels as well as higher prevalence of CVDs. Thus, stability of FBG was especially important in these participants. Results of our study strongly underscored 
Table 2 The association between FBG variability and lower-extremity PAD

\begin{tabular}{|c|c|c|c|c|c|c|}
\hline & \multicolumn{2}{|l|}{ Model 1} & \multicolumn{2}{|l|}{ Model 2} & \multicolumn{2}{|l|}{ Model 3} \\
\hline & HR (95\% Cl) & $P$ value & HR $(95 \% \mathrm{Cl})$ & $P$ value & HR $(95 \% \mathrm{Cl})$ & $P$ value \\
\hline \multicolumn{7}{|c|}{ FBG variability as continuous variable } \\
\hline Per 1 CV increase $†$ & $1.068(1.051$ to 1.08$)$ & $<0.0001$ & 1.03 (1.016 to 1.043$)$ & $<0.0001$ & 1.015 (1.001 to 1.03$)$ & 0.022 \\
\hline VIM & 1.039 (1.011 to 1.06$)$ & 0.006 & 1.037 (1.01 to 1.066$)$ & 0.007 & $1.032(1.004$ to 1.06$)$ & 0.023 \\
\hline \multicolumn{7}{|c|}{ FBG variability as categorical variable } \\
\hline \multicolumn{7}{|l|}{ SD } \\
\hline Q1 & Ref & Ref & Ref & Ref & Ref & ref \\
\hline Q2 & 1.194 (0.918 to 1.552$)$ & 0.186 & $1.189(0.914$ to 1.546$)$ & 0.196 & $1.183(0.909$ to 1.538$)$ & 0.211 \\
\hline Q3 & 1.097 (0.84 to 1.432$)$ & 0.497 & 1.088 (0.833 to 1.42$)$ & 0.536 & $1.062(0.813$ to 1.387$)$ & 0.657 \\
\hline Q1 & Ref & Ref & Ref & Ref & Ref & ref \\
\hline Q2 & 1.351 (1.041 to 1.753$)$ & 0.024 & $1.353(1.043$ to 1.757$)$ & 0.023 & 1.331 (1.025 to 1.728$)$ & 0.032 \\
\hline Q3 & $1.153(0.881$ to 1.51$)$ & 0.3 & $1.142(0.872$ to 1.497$)$ & 0.333 & $1.119(0.854$ to 1.466$)$ & 0.416 \\
\hline Q4 & 1.746 (1.357 to 2.24$)$ & $<0.0001$ & 1.682 (1.305 to 2.168$)$ & $<0.0001$ & 1.499 (1.16 to 1.938$)$ & 0.002 \\
\hline$P$ for trend & $<0.0001$ & & 0.001 & & 0.011 & \\
\hline \multicolumn{7}{|l|}{ VIM } \\
\hline Q1 & Ref & Ref & Ref & Ref & Ref & ref \\
\hline Q2 & 1.309 (1.014 to 1.69$)$ & 0.039 & 1.291 (1.0 to 1.667$)$ & 0.05 & 1.268 (0.982 to 1.638$)$ & 0.069 \\
\hline Q3 & 1.27 (0.982 to 1.643$)$ & 0.069 & 1.27 (0.99 to 1.656$)$ & 0.06 & $1.261(0.974$ to 1.632$)$ & 0.078 \\
\hline Q3 & 1.333 (1.036 to 1.715$)$ & 0.025 & $1.325(0.872$ to 1.497$)$ & 0.029 & 1.298 (1.008 to 1.672$)$ & 0.043 \\
\hline Q4 & 1.665 (1.299 to 2.136$)$ & $<0.0001$ & 1.493 (1.305 to 2.168$)$ & 0.002 & 1.419 (1.097 to 1.835$)$ & 0.008 \\
\hline $\mathrm{P}$ for trend & $<0.0001$ & & $<0.0001$ & & 0.003 & \\
\hline
\end{tabular}

Model 1 adjusted for age, race, gender, smoking and drinking status, education level.

Model 2 further adjusted for BMI, SBP, DBP, TC, TG, HDL, LDL, baseline FBG and eGFR.

Model 3 further adjusted for prevalent CHD, stroke and HT, and medication use.

${ }^{*}$ One unit increase in SD corresponds to $0.7 \%$ increment of PAD risk.

†One unit increase in CV corresponds to $1.5 \%$ increment of PAD risk.

ARV, average real variability; BMI, body mass index; CHD, coronary heart disease; CV, coefficient of variation; DBP, blood pressure; eGFR, estimated glomerular filtration rate; FBG, fasting blood glucose; HDL-C, high-density lipoprotein cholesterol; HT, hypertension; LDL-C, low-density lipoprotein cholesterol; PAD, peripheral artery disease; SBP, systolic blood pressure; TC, total cholesterol; TG, triglycerides; VIM, variability independent of the mean.

the importance of maintaining blood glucose at stable levels throughout the life course.

In a large population (>3.6 million), Savji et $a l^{29}$ concluded that lower-extremity PAD prevalence increased significantly with age. Subgroups analyses in our study demonstrated that the relation was obvious in both young and older, male and female, as well as white and black. We further stratified people with prediabetes status according to baseline FBG levels. However, results from subgroup analyses were similar among participants with or without prediabetes ( $\mathrm{p}=0.77$ for interaction). Previous studies showed that CVD risk was significantly increased in prediabetes people. ${ }^{30}{ }^{31}$ A systematic review conducted by Ford $e t a l^{2}$ showed that impaired fasting glucose (IFG) was associated with increased risk for CVD. However, there were only 3029 prediabetes participants in this study, and most of these previous studies were based on just one-time measurement of FBG. Indeed, 

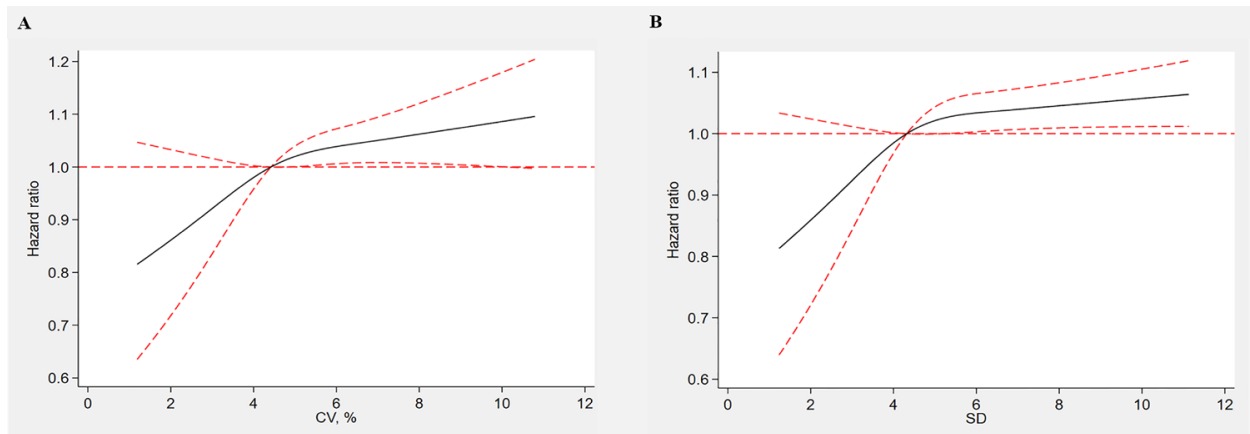

Figure 1 HRs for peripheral artery disease by fasting blood glucose (FBG) variability. The curves in (A) represent adjusted HRs (solid red line) and their $95 \% \mathrm{Cls}$ (dashed red line) based on restricted quadratic splines of FBG coefficient of variation (CV) with knots at the 5th, 50th and 95th percentiles of its distribution. The curves in (B) represent adjusted HRs of FBG SD.

compared with previous studies, our present study investigated long-term FBG variability (instead of just baseline FBG measurement) and incident lower-extremity PAD. Preiss et al investigated 6447 males from the West of Scotland Coronary Prevention Study, and followed for 15 years, found that higher FPG in people without diabetes was not associated with long-term risk of cardiovascular events. ${ }^{33}$ This population was similar to our included participants, and their results were broadly consistent with our own. Moreover, ARIC had one of the longest follow-ups of any studies, representing one of the most powerful prospective studies.

Current cardiovascular risk models differ in the end points and risk factors they consider in their development. The 2013 American College of Cardiology/American Heart Association Guideline on the Assessment of Cardiovascular Risk recommended pooled cohort equation to assess 10-year CVD risk. DM status instead of glucose level was included in this risk prediction model. ${ }^{34}$ However, two widely used risk models (QRISK2 and 5-year Framingham Risk Score) considered blood glucose levels in their prediction models. ${ }^{35} \mathrm{~A}$ few studies demonstrated that prediabetes as well as glucose levels within the normoglycemic range were independently associated with CVD and ischemic stroke. ${ }^{3336}$ Our findings further showed that FBG changes among normal range were associated with lower-extremity PAD. Fasting plasma glucose may help in identifying apparently healthy persons with early metabolic abnormalities before progression to prediabetes, and should be considered in CV risk prediction model.

Several mechanisms may be involved in the observed relation between glycemic variability and the increased risk of lower-extremity PAD. To start with, previous studies have shown that fluctuation of glycemia could trigger endothelial dysfunction, and cause oxidative stress. ${ }^{37-40}$ Also, 'glycemia swings' may enhance activation of inflammatory cytokines. ${ }^{41} 42$ Previous study also illustrated glycemic oscillations could cause apoptosis of pancreatic $\beta$-cells. ${ }^{43}$ In addition, the 'blood glucose memory' may play an important role in patients with high FBG variability in the process of lower-extremity PAD. ${ }^{44}$ Lastly, participants with high variability of FBG tended to have increased prevalence of traditional risk factors for lowerextremity PAD, including older, higher baseline FBG, BP and BMI levels. Future studies are needed to elucidate the exact mechanisms underlying the association between glycemic variability and lower-extremity PAD.

Our study had several strengths. To start with, participants included in our study were from the ARIC study. The ARIC study is a well-designed prospective study with long-term follow-up in a large population, giving us the ability to observe health trends over 25 years. Second,
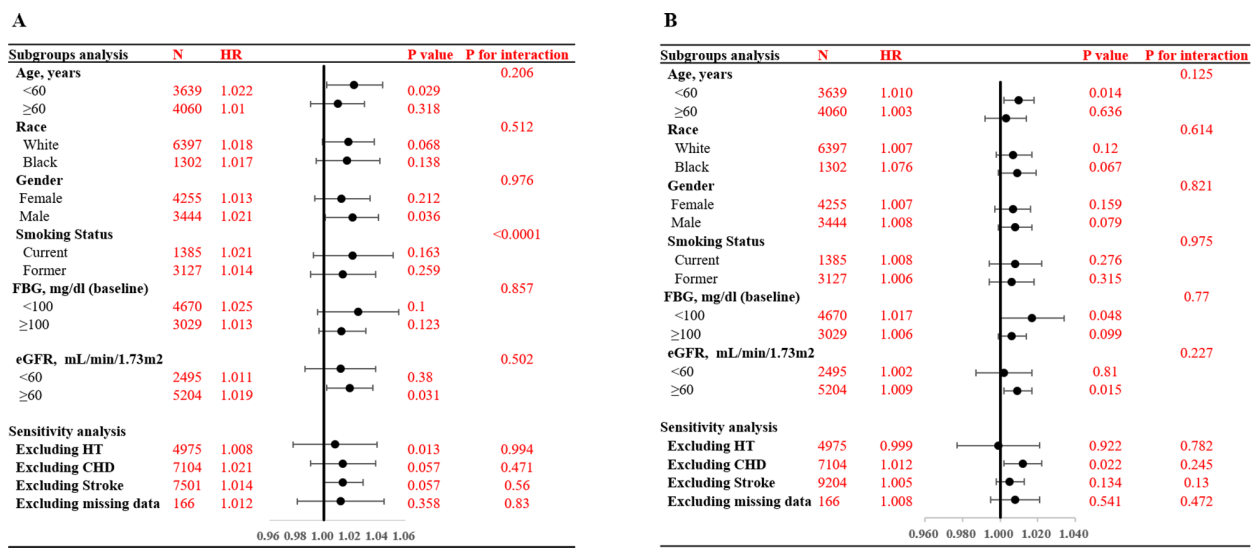

Figure 2 HR of peripheral artery disease and FBG CV (A) and SD (B) in demographic and clinical subgroups. CHD, coronary heart disease; CV, coefficient of variation; eGFR: estimated glomerular filtration rate; FBG, fasting blood glucose; HT, hypertension. 
our study included several measures of long-term FBG variability, such as CV, VIM and ARV. In addition, to our knowledge, this was the first study to demonstrate the relation between FBG variability and lower-extremity PAD. Our findings underlined that long-term chronic changes of glycemia in people without diabetes caused damage to vascular system among people without diabetes, especially those at high risk for lower-extremity PAD. Targeting these upstream risk factors could prevent lower-extremity PAD. Our findings were useful to better understand the pathogenesis of lower-extremity PAD and develop preventive strategies. Third, risk prediction models may be improved by including fasting glucose as a continuous risk factor, rather than diagnosis of diabetes.

A few limitations should be mentioned in the study. First of all, our study was not a random clinical trial. We used data from the ARIC study, so our findings needed to be confirmed in future studies. Second, ABI was measured in only a randomly sample of participants at visits 3,4 and 5. ABI was measured in only one leg at visits 3 and 4. Measurements of $\mathrm{ABI}$ in only one leg may not be able to fully diagnose lower-extremity PAD. Third, there were only three measurements of FBG in our study, our results may be can only be extrapolated to other populations without diabetes with the same number of measurements. Furthermore, we did not investigate the relationship between changes of HbAlc (a good indicator reflecting FBG variability) over time with lowerextremity PAD in the current study. As HbAlc was only measured at visits 2 and 5 in a small sample of the ARIC participants, it was difficult for us to investigate the relation between $\mathrm{HbA1c}$ variability and lower-extremity PAD. Lastly, this was an observational study, although several other major risk factors were adjusted, we cannot eliminate the possibility of residual confounding.

\section{CONCLUSIONS}

In conclusion, our study suggested that greater FBG variability was associated with an increased risk of lowerextremity PAD among people without diabetes. Glycemic change in people without diabetes should be emphasized in those with high lower-extremity PAD risk. Future clinical and basic studies are needed to confirm our findings and elucidate the exact mechanisms underlying the relation between glycemic variability and lower-extremity PAD.

Acknowledgements The authors would like to thank the staff and participants of the ARIC study for their contributions.

Contributors XTS, CZ, XDZ and XXL: research idea and study design. XTS, HMZ, ZYX, XDZ: data analysis/interpretation. XDZ, XBZ, XTS, DYY: statistical analysis. $X T S, S Z Z, Y G$ and XDZ: manuscript drafting. Each author contributed important intellectual content during manuscript writing or revision, and all authors read and approved the final manuscript.

Funding This study was supported by the National Natural Science Foundation of China (grant no:: 81870195) to XXL, and the National Natural Science Foundation of China (grant no.: 81600206) to XDZ.

Competing interests None declared.

\section{Patient consent for publication Obtained.}

Ethics approval All participants gave written informed consent prior data collection. The ARIC investigators have full ethical approval from the NIH.

Provenance and peer review Not commissioned; externally peer reviewed.

Data availability statement Data are available on reasonable request. Data may be obtained from a third party and are not publicly available. The datasets generated and/or analyzed during the current study are publicly available. The ARIC data used for this analysis are publicly available.

Supplemental material This content has been supplied by the author(s). It has not been vetted by BMJ Publishing Group Limited (BMJ) and may not have been peer-reviewed. Any opinions or recommendations discussed are solely those of the author(s) and are not endorsed by BMJ. BMJ disclaims all liability and responsibility arising from any reliance placed on the content. Where the content includes any translated material, BMJ does not warrant the accuracy and reliability of the translations (including but not limited to local regulations, clinical guidelines, terminology, drug names and drug dosages), and is not responsible for any error and/or omissions arising from translation and adaptation or otherwise.

Open access This is an open access article distributed in accordance with the Creative Commons Attribution Non Commercial (CC BY-NC 4.0) license, which permits others to distribute, remix, adapt, build upon this work non-commercially, and license their derivative works on different terms, provided the original work is properly cited, appropriate credit is given, any changes made indicated, and the use is non-commercial. See: http://creativecommons.org/licenses/by-nc/4.0/.

\section{ORCID iD}

Xiu-Ting Sun http://orcid.org/0000-0001-6677-6694

\section{REFERENCES}

1 Fowkes FGR, Rudan D, Rudan I, et al. Comparison of global estimates of prevalence and risk factors for peripheral artery disease in 2000 and 2010: a systematic review and analysis. Lancet 2013;382:1329-40.

2 Rapsomaniki E, Timmis A, George J, et al. Blood pressure and incidence of twelve cardiovascular diseases: lifetime risks, healthy life-years lost, and age-specific associations in 1.25 million people. Lancet 2014;383:1899-911.

3 Wijeysundera HC, Machado M, Farahati F, et al. Association of temporal trends in risk factors and treatment uptake with coronary heart disease mortality, 1994-2005. JAMA 2010;303:1841-7.

4 Yusuf S, Rangarajan S, Teo K, et al. Cardiovascular risk and events in 17 low-, middle-, and high-income countries. $N$ Engl J Med 2014;371:818-27.

5 Sun X, Zhuang X, Huo M, et al. Serum magnesium and the prevalence of peripheral artery disease: the Atherosclerosis risk in communities (ARIC) study. Atherosclerosis 2019;282:196-201.

6 Zhuang X, Ni A, Liao L, et al. Environment-wide association study to identify novel factors associated with peripheral arterial disease: evidence from the National health and nutrition examination survey (1999-2004). Atherosclerosis 2018;269:172-7.

7 Sarwar N, Gao P, et al, Emerging Risk Factors Collaboration. Diabetes mellitus, fasting blood glucose concentration, and risk of vascular disease: a collaborative meta-analysis of 102 prospective studies. Lancet 2010;375:2215-22.

8 Perreault L, Pan Q, Mather KJ, et al. Effect of regression from prediabetes to normal glucose regulation on long-term reduction in diabetes risk: results from the diabetes prevention program outcomes study. Lancet 2012;379:2243-51.

9 Twisk JW, Kemper HC, Mellenbergh GJ. Mathematical and analytical aspects of tracking. Epidemiol Rev 1994;16:165-83.

10 Ghouse J, Skov MW, Kanters JK, et al. Visit-to-Visit Variability of Hemoglobin $A_{1 c}$ in People Without Diabetes and Risk of Major Adverse Cardiovascular Events and All-Cause Mortality. Diabetes Care 2019;42:134-41.

11 Echouffo-Tcheugui JB, Zhao S, Brock G, et al. Visit-to-Visit glycemic variability and risks of cardiovascular events and all-cause mortality: the ALLHAT study. Diabetes Care 2019;42:486-93.

12 Xiong Z, Li J, Zhong X, et al. Visit-to-Visit fasting glucose variability in young adulthood and hippocampal integrity and volume at midlife. Diabetes Care 2019;42:2334-7.

13 Lee JH, Kim HC, Kang DR, et al. The 23-year tracking of blood lipids from adolescence to adulthood in Korea: the Kangwha study. Lipids Health Dis 2017;16:221.

14 Lyssenko V, Almgren P, Anevski D, et al. Predictors of and longitudinal changes in insulin sensitivity and secretion preceding onset of type 2 diabetes. Diabetes 2005;54:166-74. 
15 Petruski-Ivleva N, Viera AJ, Shimbo D, et al. Longitudinal patterns of change in systolic blood pressure and incidence of cardiovascular disease: the Atherosclerosis risk in Communities study. Hypertension 2016;67:1150-6.

16 Ulmer H, Kelleher C, Diem G, et al. Long-term tracking of cardiovascular risk factors among men and women in a large population-based health system: the Vorarlberg Health Monitoring \& Promotion Programme. Eur Heart J 2003;24:1004-13.

17 Norby FL, Soliman EZ, Chen LY, et al. Trajectories of cardiovascular risk factors and incidence of atrial fibrillation over a 25-year followup: the ARIC study (atherosclerosis risk in communities). Circulation 2016;134:599-610.

18 investigators TA. The Atherosclerosis risk in communities (ARIC) study: design and objectives. The ARIC Investigators. Am J Epidemiol 1989;129:687-702.

19 Muntner P, Whittle J, Lynch Al, et al. Visit-to-Visit variability of blood pressure and coronary heart disease, stroke, heart failure, and mortality: a cohort study. Ann Intern Med 2015;163:329-38.

20 Mena L, Pintos S, Queipo NV, et al. A reliable index for the prognostic significance of blood pressure variability. J Hypertens 2005;23:505-11.

21 Bangalore S, Fayyad R, Laskey R, et al. Body-Weight fluctuations and outcomes in coronary disease. $N$ Engl J Med 2017:376:1332-40.

22 Weatherley BD, Chambless LE, Heiss G, et al. The reliability of the Ankle-brachial index in the Atherosclerosis risk in communities (ARIC) study and the NHLBI family heart study (FHS). BMC Cardiovasc Disord 2006;6:7.

23 Levey AS, Bosch JP, Lewis JB, et al. A more accurate method to estimate glomerular filtration rate from serum creatinine: a new prediction equation. Modification of Diet in Renal Disease Study Group. Ann Intern Med 1999;130:461-70.

24 Emerging Risk Factors Collaboration, Sarwar N, Gao P, et al. Diabetes mellitus, fasting blood glucose concentration, and risk of vascular disease: a collaborative meta-analysis of 102 prospective studies. Lancet 2010;375:2215-22.

25 Selvin E, Steffes MW, Zhu H, et al. Glycated hemoglobin, diabetes, and cardiovascular risk in nondiabetic adults. $N$ Engl J Med 2010;362:800-11.

26 Lawes CMM, Parag V, Bennett DA, et al. Blood glucose and risk of cardiovascular disease in the Asia Pacific region. Diabetes Care 2004;27:2836-42.

27 Hirakawa $\mathrm{Y}$, Arima $\mathrm{H}$, Zoungas S, et al. Impact of visit-to-visit glycemic variability on the risks of macrovascular and microvascular events and all-cause mortality in type 2 diabetes: the advance trial. Diabetes Care 2014;37:2359-65.

28 Bancks MP, Carnethon MR, Jacobs DR, et al. Fasting glucose variability in young adulthood and cognitive function in middle age: the coronary artery risk development in young adults (cardia) study. Diabetes Care 2018;41:2579-85.

29 Savji N, Rockman CB, Skolnick AH, et al. Association between advanced age and vascular disease in different arterial territories: a population database of over 3.6 million subjects. J Am Coll Cardiol 2013;61:1736-43.

30 Hubbard D, Colantonio LD, Tanner RM, et al. Prediabetes and risk for cardiovascular disease by hypertension status in black adults: the Jackson heart study. Diabetes Care 2019;42:2322-9.

31 Vistisen D, Witte DR, Brunner EJ, et al. Risk of cardiovascular disease and death in individuals with prediabetes defined by different criteria: the Whitehall II study. Diabetes Care 2018;41:899-906.

32 Ford ES, Zhao G, Li C. Pre-Diabetes and the risk for cardiovascular disease: a systematic review of the evidence. J Am Coll Cardiol 2010;55:1310-7.

33 Preiss D, Welsh P, Murray HM, et al. Fasting plasma glucose in nondiabetic participants and the risk for incident cardiovascular events, diabetes, and mortality: results from WOSCOPS 15-year follow-up. Eur Heart J 2010;31:1230-6.

34 Goff DC, Lloyd-Jones DM, Bennett G, et al. 2013 ACC/AHA guideline on the assessment of cardiovascular risk: a report of the American College of Cardiology/American heart association Task force on practice guidelines. Circulation 2014;129:S49-73.

35 Khanji MY, Bicalho VVS, van Waardhuizen CN, et al. Cardiovascular risk assessment: a systematic review of guidelines. Ann Intern Med 2016;165:713-22.

36 Sung J, Song Y-M, Ebrahim S, et al. Fasting blood glucose and the risk of stroke and myocardial infarction. Circulation 2009;119:812-9.

37 Eelen G, de Zeeuw P, Treps L, et al. Endothelial cell metabolism. Physiol Rev 2018;98:3-58.

38 Brownlee M, Hirsch IB. Glycemic variability: a hemoglobin A1c-independent risk factor for diabetic complications. JAMA 2006;295:1707-8.

39 Innat MA, Thorpe JE, Kamat CD, et al. Reactive oxygen species mediate a cellular 'memory' of high glucose stress signalling. Diabetologia 2007;50:1523-31.

40 Ceriello A, Esposito K, Piconi L, et al. Oscillating glucose is more deleterious to endothelial function and oxidative stress than mean glucose in normal and type 2 diabetic patients. Diabetes 2008;57:1349-54.

41 Esposito K, Nappo F, Marfella R, et al. Inflammatory cytokine concentrations are acutely increased by hyperglycemia in humans: role of oxidative stress. Circulation 2002;106:2067-72.

42 Horváth EM, Benko R, Kiss L, et al. Rapid 'glycaemic swings' induce nitrosative stress, activate poly(ADP-ribose) polymerase and impair endothelial function in a rat model of diabetes mellitus. Diabetologia 2009;52:952-61.

43 Del Guerra S, Grupillo M, Masini M, et al. Gliclazide protects human islet beta-cells from apoptosis induced by intermittent high glucose. Diabetes Metab Res Rev 2007;23:234-8.

44 Keating ST, El-Osta A. Glycemic memories and the epigenetic component of diabetic nephropathy. Curr Diab Rep 2013;13:574-81. 